\title{
Response to invited commentary: "Event-based versus process-based informed consent to address scientific evidence and uncertainties in ionizing medical imaging" by Recchia et al
}

\author{
Virginia Recchia $\cdot$ Antonio Dodaro $\cdot$ Larissa Braga
}

Received: 7 August 2013 / Accepted: 12 August 2013 /Published online: 10 September 2013

(C) The Author(s) 2013. This article is published with open access at Springerlink.com

We thank Prof. Vock for his noteworthy and constructive commentary [1]. This gives us the opportunity to briefly provide additional observations to elaborate this topic further. We agree with Prof. Vock that our theoretical approach [2] may not be applied in a non-invasive and low-dose medical imaging. Nevertheless, this makes the innovation that we are trying to develop even more challenging. We are all discussing about something similar to a "paradigm shift", in the sense described by Kuhn [3]: (a) presenting results "sufficiently unprecedented to attract an enduring group of adherents away from competing modes of scientific activity" and (b) "being sufficiently open-ended to leave all sorts of problems for the redefined group of practitioners to solve."

\section{Who is responsible for obtaining informed consent?}

This question could be completely answered only if contextualised in each legal State taken into consideration, which is clearly beyond the scope of our article. We agree, however, with Prof. Vock that in the informed consent process in medical imaging there is a range of factual situations in need to be optimised in terms of coordination between the

This reply refers to the comment available at doi:10.1007/s13244-013-0283-3

\section{Recchia $(\bowtie) \cdot$ A. Dodaro}

Health Technology Assessment, Epidemiology and Communication, CNR-IFC Italian National Research Council-Institute of Clinical Physiology, c/o Ecotekne University Campus, Prov.le

Lecce-Monteroni, 73100 Lecce, Italy

e-mail: recchia@ifc.cnr.it

L. Braga

Research on Research Organization, Duke Medical Center, Durham, $\mathrm{NC}$, USA referring and executing physicians. Risk communication and informed consent do not represent an exclusive competence of the referrer, as ethically, legally and clinically attributable even to the radiologist. Therefore, it seems unconceivable to rule out a direct communicative duty in informed consent, as well as the possibility of a refusal to perform the examination if deemed inappropriate. As a result, the education and training of professionals should focus not only on radiation protection but also on risk communication and informed consent $[4,5]$.

\section{Is informed consent identical for all imaging examinations using ionising radiation?}

At a level of abstraction, informed consent should be identical for all imaging examinations using ionising radiation. From a legal and ethical viewpoint, the patient's will deserves to be expressed in full, with freedom and real awareness; and it is precisely in this regard that we find, in a nutshell, the essence of informed consent. Nonetheless, we are in line with Prof. Vock that in front of this mandatory statement it is necessary to develop a consistent fine-tuning of the ideal model, accordingly with the different peculiarities of the real clinical practice.

\section{Is there a threshold for the need of risk information?}

At the level of risk acceptability, we have to put together too many variables only to determine the cases in which informed consent is necessary. We have to contemplate both the variables involved in the personalised risk (dose, age, sex, genetic variables, etc.) and the variables that determine the acceptability by the specific patient. Moreover, if we consider that an average acceptable risk is 1:2,000, we assume that the patient 
has an average risk propensity, while we know that there are many variables that affect his willingness to take risks.

Complicating this picture, even healthcare professionals have different levels of risk propensity that inevitably come into play in medical decision-making. Consequently, in order to decide whether to make an explicit and comprehensive informed consent, to ex ante consider individual acceptability may not be the right way. In a sense, there would be once again too many filters to the final choice of the patient. Instead, if the filter is given only by the dose threshold (and perhaps age), the patient can, based on his subjective characteristics, decide freely and consciously if his personalised risk is acceptable and whether or not to undergo the imaging exam, even considering the proposed alternatives $[5,6]$.

\section{How do we communicate the risk and stochastic uncertainties?}

If we deem it too difficult to communicate radiation risk in medical imaging, we should not communicate it at all. Why would we only communicate the average population risk and omit the personalised risk? Conversely, if we follow the logic that it is right to provide a patient with the most accurate information possible, then the patient can make the decision he or she believes to be the most acceptable. We should strive to create conditions so that the needed information could be conveyed also through a personalised risk communication. In this way, a real integrated process of informed consent would be merely the most natural, proper and routine approach to protect both physician and patient in decision-making. In this way, we believe that we all—as a scientific communitywould be acting to find possible solutions to what looks like a new paradigm.

Conflicts of interest The authors declare no conflicts of interest. No funding was received for this work.

Open Access This article is distributed under the terms of the Creative Commons Attribution License which permits any use, distribution, and reproduction in any medium, provided the original author(s) and the source are credited.

\section{References}

1. Vock P (2013) Invited Commentary: "Event-based versus processbased informed consent to address scientific evidence and uncertainties in ionising medical imaging" by Recchia et al. Insights Imaging. doi:10.1007/s13244-013-0283-3

2. Recchia V, Dodaro A, Braga L (2013) Event-based versus processbased informed consent to address scientific evidence and uncertainties in ionising medical imaging. Insights Imaging. doi:10.1007/ s13244-013-0272-6

3. Kuhn TS (1970) The Structure of Scientific Revolutions, 2nd edn. University of Chicago Press, Chicago

4. Consumers Health Forum of Australia (2013) Diagnostic Imaging and Informed Consent: A Literature and Research Scoping Paper. February. https://www.chf.org.au/pdfs/rep/rep-1027-DI-scoping-paper.pdf. Accessed 02 Aug 2013

5. Picano E (2004) Informed consent and communication of risk from radiological and nuclear medicine examinations: how to escape from a communication inferno. BMJ 329(7470):849-51

6. Supreme Court of Wisconsin (2012) WI 39 - Case No.: 2008AP1972. http://www.wicourts.gov/sc/opinion/DisplayDocument.pdf?content= pdf\&seqNo=81164. Accessed 01 Aug 2013 